\title{
AE-D Measurements of the NO Geomagnetic Latitudinal Distribution and Contamination by $\mathrm{N}^{+}\left({ }^{5} S\right)$ Emission
}

\author{
J. C. Gérard and C. E. NoËL \\ Institut d'Astrophysique, Université de Liège, Liège-Ougrée, Belgium
}

\begin{abstract}
Measurements of the NO $\gamma(1,0)$ band resonance scattering distribution obtained with the Atmosphere Explorer D satellite have been inverted to generate maps of the meridional distribution of nitric oxide between 90 and $160 \mathrm{~km}$. It is shown that the results are better organized in terms of geomagnetic rather than geographic latitudes, especially at high latitudes. In particular, the presence of an $E$ region maximum associated with the summer auroral zone is clearly demonstrated. The existence below the NO peak of larger concentrations in the winter than in the summer hemisphere is also observed. Comparison between calculations and rocket observations of the $2143-\AA$ doublet emission indicates that the quantum yield of $\mathrm{N}^{+}\left({ }^{5} S\right)$ is considerably less than unity. The possible contamination of the observations by this emission is shown to be negligible in these observations.
\end{abstract}

\section{INTRODUCTION}

Satellite observations of the fluorescence of the nitric oxide sands have provided information about the vertical and latitudinal distribution of thermospheric NO and its dependence on geophysical factors such as season, solar activity, and local time. The NO ultraviolet spectrometer (UVNO) on the Atmosphere Explorer C and D satellites [Barth et al., 1973] in particular measured the intensity distribution of the $(1,0) \gamma$ band centered near $2149 \AA$. High-latitude observations obtained on AE-D despun orbits were analyzed by Cravens [1981], whereas Cravens et al. [1979] presented measurements at $200 \mathrm{~km}$ from AE-C despun orbits.

Data obtained with AE-D in the spinning mode were recently presented by Cravens et al. [1985]. They provide a data base describing the statistical distribution of thermospheric NO at solstice during the period of very low solar activity that prevailed during the AE-D mission. The measurements described in that paper were presented in terms of contour plots of the NO concentrations versus altitude and geographic latitude. They showed a maximum concentration near $110 \mathrm{~km}$ with a peak density minimum near $40^{\circ} \mathrm{N}$ and increasing with latitude in both hemispheres. However, a steeper gradient was observed in the northern (winter) hemisphere. Since particle precipitations are known to be responsible for the increase of the NO density in the polar regions, it is of interest to analyze the NO distribution in geomagnetic coordinates to verify the existence of a high-latitude maximum associated with the statistical location of the auroral zones.

We also investigate the question of the potential contamination of the fluorescence scattering measurements of the NO $(1,0) \gamma$ band by the $2143-\AA$ emission originating from the $\mathrm{N}^{+}\left({ }^{5} S\right)$ doublet. This emission was first observed in the spectrum of the ultraviolet aurora by Duysinx and Monfils [1972] and Sharp and Rees [1972] and later identified as the $\mathrm{N}^{+}\left({ }^{5} S\right)$ ${ }^{3} P_{2}-{ }^{5} S_{2}$ doublet by Dick [1978]. Its intensity in the ultraviolet dayglow was measured from a rocket by Barth and Steele [1982] and recently from Spacelab 1 by Torr and Torr [1985a]. Calculations by Victor and Dalgarno [1982] indicat-

Copyright 1986 by the American Geophysical Union.

Paper number 5A8288.

0148-0227/86/005A-8288\$05.00 ed that its presence in the passband of ultraviolet spectrometers may contribute to the signal assigned to the $(1,0)$ gamma band at high altitudes. It is presumably excited by electron impact dissociative ionization in the aurora and dissociative ionization by photons with energy above $329 \AA$ in the dayglow. We examine whether contamination of the present AE-D NO gamma measurements by the N II 2143-Å dayglow emission is significant.

\section{Inversion of the Vertical Profiles}

The data set analyzed in this study is identical to that used by Cravens et al. [1985] to investigate the distribution of the NO horizontal column density. Its characteristics were described by Cravens et al. [1985] and will only be summarized below. The measurements were collected on AE-D spinning orbits from late November 1975 to early February 1976. The local time of the perigee shifted approximately from 1000 at $70^{\circ} \mathrm{N}$ to 0530 at the South Pole. In order to preserve a vertical resolution better than $10 \mathrm{~km}$ at the NO peak and to avoid any significant contamination by the $\mathrm{N}^{+}\left({ }^{5} S\right)$ doublet, only data collected when the spacecraft was between $200 \mathrm{~km}$ and perigee were considered in this study.

In order to improve the statistics, three consecutive spins were added together to form a vertical profile. Data points were subsequently filtered out, averaged, or rejected, depending on the quality of the data. The Rayleigh scattering contribution was removed by fitting an exponentially decreasing curve to the signal measured at a tangent ray height of $75 \mathrm{~km}$. Figure 1 shows an example of a vertical NO profile measured at low latitudes. The total number of counts, the subtracted Rayleigh scattering, and the resulting difference are plotted as a function of the altitude of the minimum ray height. In this profile the Rayleigh scattering contribution becomes negligible above $120 \mathrm{~km}$ and reaches about $25 \%$ of the total signal near $100 \mathrm{~km}$. The NO and the Rayleigh scattering contributions are equal at $90 \mathrm{~km}$, and at $80 \mathrm{~km}$, virtually all the signal is due to Rayleigh scattering. The relative magnitude of the two contributions depends on the NO amount and the solar zenith angle. In the most unfavorable case the Rayleigh scattering signal contributes about $50 \%$ of the total of the NO peak. The intersection of the Rayleigh scattering and corrected NO signal is always located between 90 and $95 \mathrm{~km}$. The Rayleigh scattering contribution remains below $1 \%$ above $120 \mathrm{~km}$. An- 


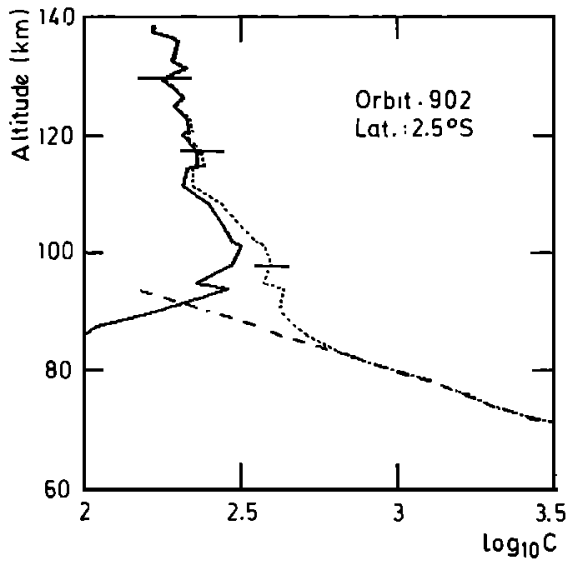

Fig. 1. Plot of the decimal logarithm of the counting rate (counts/21 ms) measured on a low-latitude profile by the UVNO experiment. The dotted line shows the total signal, the dashed line is the subtracted Rayleigh scattering contribution, and the solid line represents the difference. The error bars indicate the standard deviation of the count rate.

other effect of the removal of the Rayleigh scattering contribution is to slightly increase the altitude of the NO maximum compared to the uncorrected profiles.

Counts were then converted into NO horizontal column densities by combining the curve of growth of the NO $\gamma(1,0)$ emission rate with the instrumental sensitivity. Finally, a total of 1325 profiles of the NO horizontal column versus altitude of the tangent point entered the data set.

As the line of sight of the telescope scans the limb below the spacecraft, a series of column emission rates $4 \pi I$ are obtained. They are related to the NO density by the relationship

$$
4 \pi I(z)=10^{-6} g \int_{-\infty}^{\infty} n(s) d s
$$

where $d s$ is the element along the line of sight, $n$ the local NO concentration, and $g$ the emission rate factor equal to 7.7 $\times 10^{-6} \mathrm{~s}^{-1}$ for the optically thin $(1,0)$ NO $\gamma$ band. Because of possible self-absorption, $g$ depends on the NO column density between the sun, the scattering volume, and the satellite. The relationship given by Stewart and Cravens [1978], corrected for the AE-D instrument sensitivity, is adopted.

If the spacecraft altitude is substantially larger than the tangent ray $p$ and assuming spherical symmetry, equation (1) may be written in the form of a Fredholm integral equation of the first kind:

$$
4 \pi I(p)=2 \times 10^{-6} g \int_{p}^{\infty} \frac{n(r) r}{\left(r^{2}-p^{2}\right)^{1 / 2}} d r
$$

where $n(r)$ is the geocentric NO vertical distribution and $r$ the radial distance.

Various numerical methods (substitution, least squares approximation, iterative) may be used to invert equation (2). However, this relation is an Abel integral equation which has an analytical solution given by

$$
n(r)=-\frac{1}{2 \times 10^{-6} g \pi} \frac{d}{d r} \int_{r}^{\infty} \frac{4 \pi I(p)}{\left(p^{2}-r^{2}\right)^{1 / 2}} \frac{r}{p} d p
$$

Roble and Hays [1972] gave a method to solve (3) numeri- cally. Equation (3) is first written in the equivalent form;

$$
n(r)=-\frac{1}{2 \times 10^{-6} g \pi} \int_{r}^{\infty} \frac{d(4 \pi \mathrm{I}) / d p}{\left(p^{2}-r^{2}\right)^{1 / 2}} d p
$$

A function $f(p)$ representing $4 \pi I$ is then locally fitted to the measurements over $N$ adjacent data points using a least squares method. The derivative inside integral (4) may then be obtained analytically, and the integral is replaced by a discrete sum. In the case of the AE-D data a quadratic form was used for $f(p)$, and the smoothing parameter $N$ was taken equal to 5 , a compromise between statistical noise smoothing and spatial resolution.

\section{Latitudinal Distribution}

The Abel inversion (4) using the numerical procedure described above was applied to all the individual intensity profiles of the data set. The resulting NO density data points were then sorted into $5^{\circ}$-latitude by $2-\mathrm{km}$-altitude bins. The data in each bin were averaged, and a contour map of NO concentrations was constructed with the inverted data. The distribution versus geographic latitude is shown in Figure 2. As expected, it is very similar to the map in Figure 14 by Cravens et al. [1985], although the inversion was made using a different method. It shows a clear latitudinal NO gradient with minimum values less then $1 \times 10^{7} \mathrm{~cm}^{-3}$ near $20^{\circ} \mathrm{N}$, rising more steeply in the northern hemisphere. Comparison with Cravens et al. [1985], however, indicates that the present number densities are somewhat less than their values. This may be explained in part by the fact that although they originate from the same global data base, the two data sets are not identical. Besides, in this work the Rayleigh scattering contribution was subtracted from the low-altitude signal, in contrast to the previous results.

Figure 3 shows the same inverted data, organized in geomagnetic latitudes. Several interesting differences from Figure 2 are observed. The most conspicuous one is seen at high summer latitudes, where a clear maximum is now obtained near $70^{\circ} \mathrm{S}$. This peak in the southern auroral zone is associated with the effects of the high-energy particle precipitations, which generate odd nitrogen in the lower thermosphere through direct and indirect $\mathrm{N}_{2}$ dissociation. This maximum, predicted by two-dimensional models [Gérard et al., 1984; Gérard and Roble, 1986], is not clearly apparent in Figure 2,

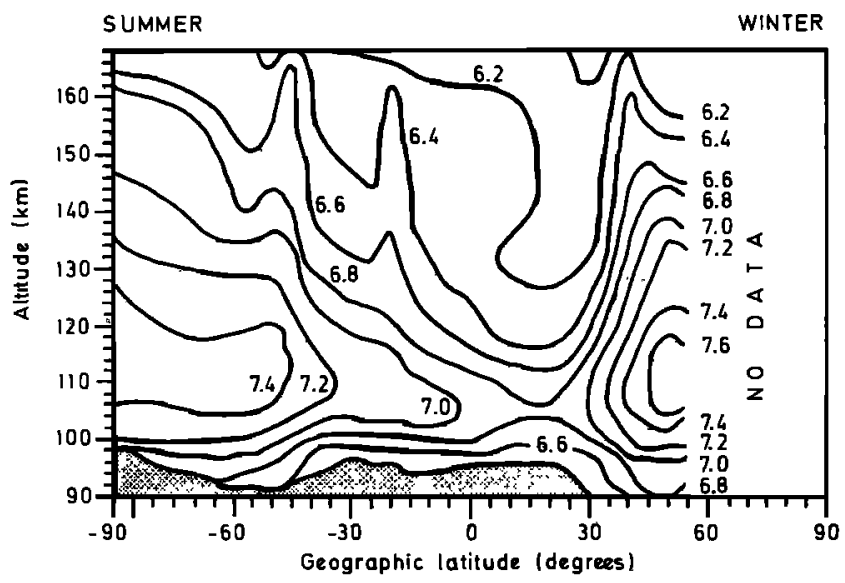

Fig. 2. Contour plot in geographic latitudes of $\log _{10}$ of the nitric oxide concentration (per cubic centimeters) obtained by inverting the UVNO measurements. 


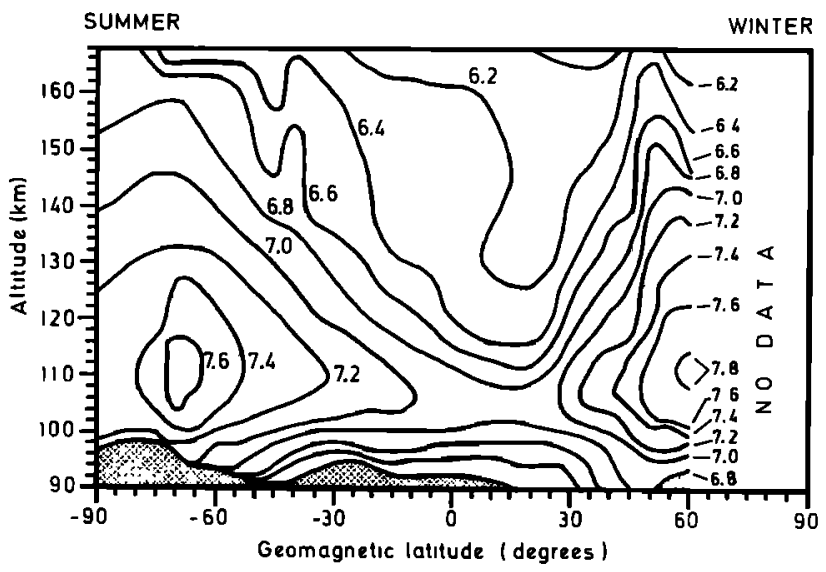

Fig. 3. Same as Figure 2 in geomagnetic latitudes.

where the latitudinal distribution is spread out by the use of geographic coordinates. It is thus clear that geomagnetic latitude is a more appropriate coordinate than geographic latitude to represent the high-latitude thermospheric NO distribution. The low-latitude part of the map is virtually unchanged. In the winter hemisphere the contours suggest that another peak may exist at latitudes higher than $60^{\circ} \mathrm{N}$. The lack of high-latitude winter data is inherent in the experimental method, which requires the observed region to be sunlit.

Another feature, common to both figures, is the large NO density below the NO peak observed at latitudes higher than $40^{\circ} \mathrm{N}$ in contrast to the low values below $100 \mathrm{~km}$ at high summer latitudes. This is also in agreement with model calculations, which predict a stronger downward flow of NO into the mesosphere in the winter than in the summer regions. It is also consistent with the trend which may be deduced from the comparison of NO rocket measurements at various latitudes [Gérard and Deneye, 1984]. It is probably a consequence of the large diurnally averaged photolysis rate of nitric oxide by solar radiation near $1980 \AA$. Another possible factor is the seasonal dependence of the strength of downward turbulent transport [Garcia and Solomon, 1985].

In addition to the natural variability of nitric oxide, the main sources of error are (1) the statistical photon noise in the limb scans, whose standard deviation is between 3 and $10 \%$ of the measured count rate, (2) the presence of stars undetected by the filtering procedure, which may erroneously increase the value of some data points, and (3) the inversion procedure itself, which introduces an error that depends on the statistical noise, the smoothing parameter $N$, and the interval between successive samples.

The variability of the derived NO density is illustrated in Figure 5, where the standard deviation of the concentration is shown for three different latitude bins at $130 \mathrm{~km}$ and at the altitude of the maximum. It is of the order of $100 \%$ at both altitudes. The standard deviation of the altitude of the peak is shown to be about $5 \mathrm{~km}$. It is clear that the natural variability of NO exceeds by far the other sources of uncertainties described above.

\section{CONTAMination by THE $\mathbf{N}^{+}\left({ }^{5} S\right)$ Emission}

Victor and Dalgarno [1982] suggested that the excitation of metastable $\mathrm{N}^{+}\left({ }^{5} S\right)$ ions in the sunlit atmosphere proceeds through the process

$$
N_{2}+h v \rightarrow \mathrm{N}^{+}\left({ }^{5} S\right)+\mathrm{N}\left({ }^{4} S\right)+e^{-}
$$

They indicated that the cross section for this process may be estimated by assuming that each removal of an electron on the $2 s \sigma_{g}$ orbital of $\mathrm{N}_{2}$ leads to the production of an $\mathrm{N}^{+}\left({ }^{5} S\right)$ ion. The excitation cross section $\sigma_{N^{+}}$for this removal may be obtained from the differential oscillator strength for removal of $2 s \sigma_{g}$ electrons measured by Wight et al. [1976] up to $50 \mathrm{eV}$. Thus the rate of $\mathrm{N}^{+}\left({ }^{5} S\right)$ ion production is given by

$$
P\left[\mathrm{~N}^{+}\left({ }^{5} \mathrm{~S}\right)\right]=\left[\mathrm{N}_{2}\right] \varepsilon \int_{0}^{\lambda_{M}} \pi F(\lambda) \sigma_{\mathrm{N}^{+}}(\lambda) d \lambda
$$

where $\varepsilon$ is the yield of $\mathrm{N}^{+}\left({ }^{5} S\right)$ ions in the $2 s \sigma_{g}$ electron removal, $\lambda_{m}$ the energy threshold equal to $329 \AA$, and $\pi F(\lambda)$ the attenuated solar irradiance. The volume emission rate $\eta$ is deduced from (6) assuming that $\mathrm{N}^{+}\left({ }^{5} S\right)$ is quenched by $\mathrm{N}_{2}$ with a deactivation coefficient $k=2.5 \times 10^{-9} \mathrm{~cm}^{3} \mathrm{~s}^{-1}$ [Knight, 1982].

A determination of the value of $\varepsilon$ may be obtained by analyzing the rocket observations by Barth and Steele [1982]. This measurement of ultraviolet dayglow was made in November 1981 for a solar zenith angle of $61.7^{\circ}$ at a latitude of $40^{\circ} \mathrm{N}$. At $200 \mathrm{~km}$ the measured emission rate was $333+67 \mathrm{R}$ at a zenith angle of $87.6^{\circ} \pm 0.5^{\circ}$. Since the EUV solar fluxes derived from Atmosphere Explorer observations were recently revised, we reanalyze the observations of Barth and Steele, carefully considering the geometry of the observations. In these calculations the solar irradiances are calculated using the revised solar fluxes recently published by Torr and Torr [1985b] and the effective absorption cross sections given by Torr et al. [1979]. The neutral atmosphere densities are obtained from the modified mass spectrometer/incoherent scatter model atmosphere [Hedin, 1983].

Using the geophysical parameters appropriate to the time of the observations $(F 10.7=235, F 10.7=216, A p=4)$ and the solar fluxes given by Torr and Torr [1985b] appropriately scaled to the value of $F 10.7$, the vertical distribution of $\eta$ is calculated and integrated from $200 \mathrm{~km}$ along the line of sight of the instrument. The calculated volume emission rate reaches a peak of $54 \mathrm{ph} / \mathrm{cm}^{3} \mathrm{~s}$ at $195 \mathrm{~km}$ (Figure 4). The integrated column emission rate is $3.65 \mathrm{kR}$ with limits of 3.5

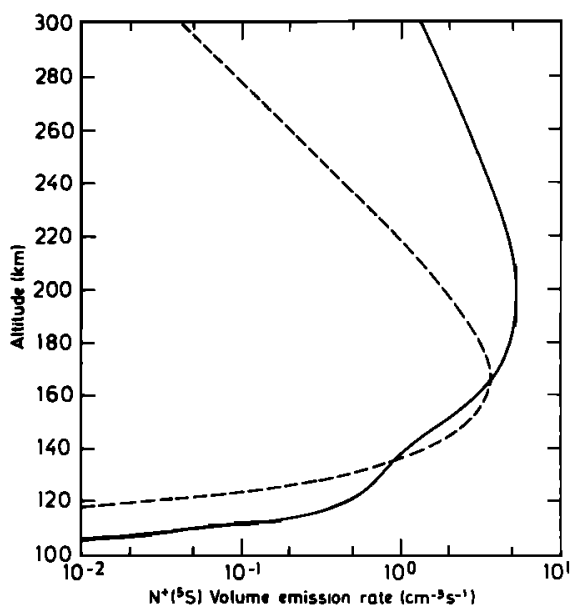

Fig. 4. Calculated vertical distribution of the N II 2143-A volume emission rate for the conditions of the Barth-Steele rocket measurements (solid line) and for the AE-D UVNO observations (dashed line). The solar zenith angle for both curves is $67.1^{\circ}$, and the $\mathrm{N}^{+}\left({ }^{5} S\right)$ yield is 0.1 (see text). 


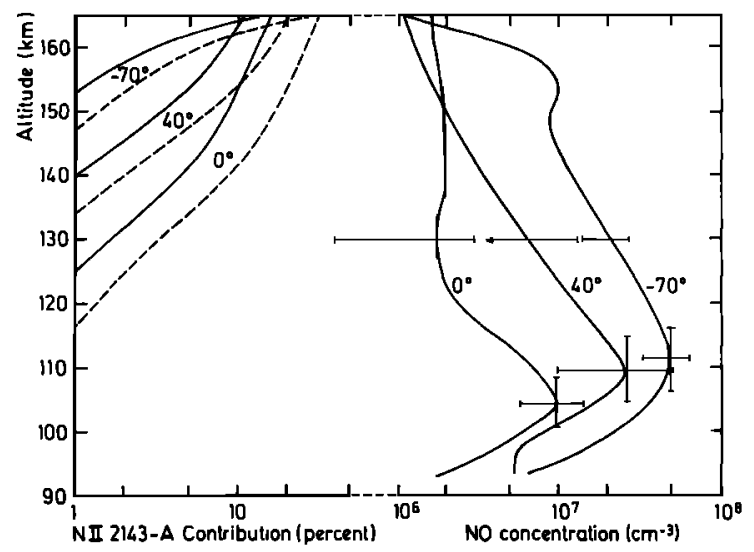

Fig. 5. Three examples of NO vertical profiles averaged over $5^{\circ}$ of latitude and corresponding calculated contamination by the $\mathrm{N}^{+}\left({ }^{5} S\right)$ doublet. The fractional contribution of the $2143-\AA$ doublet is plotted lor three different latitudes and two values of the $\mathrm{N}^{+}\left({ }^{3} S\right)$ yield $\varepsilon=0.1$ (solid lines) and 0.2 (dashed lines).

and $3.9 \mathrm{kR}$. Comparing this value to the experimental result, a value $\varepsilon=0.09$ is obtained. This value is substantially smaller than the $18 \%$ derived from the comparison of the same experimental data with the calculations by Victor and Dalgarno [1982]. The difference between the two calculations is traceable to the zero optical depth production efficiency and thus to the solar fluxes used. Values of $1.6 \times 10^{-8} \mathrm{~s}^{-1}$ for solar minimum and $2.4 \times 10^{-8} \mathrm{~s}^{-1}$ for solar maximum are given by Victor and Dalgarno, whereas our values are $1.2 \times 10^{-8}$ and $3.9 \times 10^{-8} \mathrm{~s}^{-1}$, respectively. Slightly different $\mathrm{N}_{2}$ densities and a somewhat different extrapolation toward short wavelengths of Wight et al.'s [1976] measurements may also contribute to the difference. A value of $20 \%$ was also deduced for $\varepsilon$ by Torr and Torr [1985a], based on Spacelab 1 limb scannings of the $2143-\AA$ emission made with an imaging spectrometer at a resolution of $2.5 \AA$. However, these measurements were made for unfavorable conditions near the terminator where the solar zenith angle changed by over $13^{\circ}$ during the vertical scans.

In summary, dayglow observations of the $\mathrm{N}^{+}\left({ }^{5} S\right)$ emission indicates that the yield of $\mathrm{N}^{+}\left({ }^{5} S\right)$ ions is considerably less than unity, based on the measurements of Wight et al. [1976]. Rocket and Spacelab observations give a range of values between 0.09 and 0.20 . In the following discussion a value of 0.1 will be adopted.

We now consider the AE-D NO measurements to examine whether the presence of the $\mathrm{N}^{+}\left({ }^{5} S\right)$ 2143- $\AA$ doublet makes any substantial contribution to the observations described before. Figure 4 shows the calculated volume emission rate using parameters typical of the AE-D solar minimum activity period $(A p=12, F 10.7=73)$ for a solar zenith angle of $67.1^{\circ}$. The peak emission rate reaches a value of $3.7 \mathrm{~cm}^{-3} \mathrm{~s}^{-1}$. It is located at $165 \mathrm{~km}$ and is thus lower in altitude and smaller in magnitude than at solar maximum.

Figure 5 shows the vertical profiles of NO obtained at three geomagnetic latitudes and averaged over $5^{\circ}$ of latitude. They show distinctive features such as peak concentrations, peak altitudes, and shape above and below the maximum, as evidenced in Figures 2 and 3. The vertical distributions of the $\mathbf{N}$ II $2143-\AA$ emission rate has been calculated following equation (4) as described before and using values of solar zenith angles and local times appropriate to each latitude. To esti- mate the possible contamination of the NO maps by this emission, the emission rate of $\mathrm{N}^{+}\left({ }^{5} S\right)$ is calculated and converted into fractional contribution of the UVNO signal by considering the efliciency of the instrument collection of the $\mathrm{NO}$ and $\mathrm{N}^{+}\left({ }^{5} S\right)$ emissions. The results are shown in Figure 5 for two values of the efficiency and indicate that the contamination is totally negligible in the lower thermosphere and amounts to less than $15 \%$ at higher altitudes at all latitudes. Its relative contribution maximizes at low latitudes. Even if the $\mathrm{N}^{+}\left({ }^{5} S\right.$ ) yield was 0.2 , the contribution would be less than $30 \%$ and would thus not significantly affect the derived NO densities.

Results of $\mathrm{N}^{+}\left({ }^{5} S\right)$ calculations must be considered with some caution until discrepancies between values of $\varepsilon$ deduced from the dayglow observations and laboratory measurements are understood. The peak cross section of $1.1 \times 10^{-18} \mathrm{~cm}^{-2}$ for the removal of $2 s \sigma_{g}$ electrons [Wight et al., 1976] is comparable with the value deduced from the auroral [Dalgarno $e t$ al., 1981] and laboratory [Knight, 1982] measurements, thus suggesting a high efficiency for the production of $\mathrm{N}^{+}\left({ }^{5} S\right)$ ions by electron impact. It is not clear why the efficiency would be substantially smaller in the case of photoionization. Other sources of uncertainties in the $\mathrm{N}^{+}\left({ }^{5} S\right)$ distribution include the value of the solar irradiance below $329 \AA$, its dependence on solar activity, and the vertical distribution of $\mathrm{N}_{2}$ as given by model atmospheres. More measurements of the 2143- $\AA$ doublet and comparisons with model calculations in various geophysical conditions are clearly needed to clarify this question.

\section{Discussion ANd Conclusions}

The latitudinal distribution of thermospheric nitric oxide is best represented in terms of geomagnetic rather than geographic latitudes, especially at high latitudes. The existence of a high-latitude $E$ region maximum in the vicinity of the summer auroral zone is clearly demonstrated. Even higher concentrations are probably associated with the winter auroral zone, where densities exceed $7 \times 10^{7} \mathrm{~cm}^{-3}$, whereas the low-latitude peak values are less than $1 \times 10^{7} \mathrm{~cm}^{-3}$. The reverse trend was observed by Cravens [1981] and predicted by model calculations at higher $(200 \mathrm{~km})$ altitudes, where the summer concentrations exceed the winter values. It sho: Id be remembered that these are latitudinally averaged values at quiet solstice periods very close to solar cycle minimum. Steeper latitudinal gradients are probably present at given particular times. The shape of the vertical profiles is latitude dependent, as discussed by Cravens et al. [1985]. This is clearly examplified by the drastic differences in shape among the three profiles in Figure 4.

The main features of the observed NO distribution are reproduced satisfactorily by the two-dimensional models of Gérard et al. [1984] and Gérard and Roble [1986]. In particular, the steeper latitudinal gradient in winter than summer and the maximum associated with the southern auroral zone are in agreement with the model results. This accord is improved when the data are plotted in terms of geomagnetic latitudes. The use of geographic coordinates tends to smear out the peaks associated with the zones of particle precipitation. The agreement with two-dimensional models is also improved by removing the Rayleigh scattering. Figures 2 and 3 suggest that the minimum associated with the NO photolysis near the mesopause and the weaker $K$ coefficient in summer is deeper in the summer hemisphere, in agreement with the twodimensional model results. However, a quantitative compari- 
son is made difficult by the weakness of the NO scattering compared to the Rayleigh scattering below $100 \mathrm{~km}$. The shapes of the vertical profiles and their latitudinal dependence are also well matched by the calculations.

Model calculations of the vertical distribution of the $\mathrm{N}^{+}\left({ }^{5} S\right)$ volume emission rate show that its contribution in the data analyzed here is negligible. Nevertheless, the relative importance of the $2143-\AA$ doublet depends on altitude, local time, latitude, and season as well as instrument characteristics. It also depends on solar activity, and comparison between the two curves in Figure 4 shows that it increases by over an order of magnitude at high altitudes. The solar cycle dependence of the high-altitude NO concentration has not been observed, but it may be expected that it responds differently from $\mathrm{N}^{+}\left({ }^{5} S\right)$ to changes in solar activity. Consequently, the possible contamination by the $2143-\AA$ dayglow must be evaluated carefully for each observational condition.

Acknowledgments. One of us (J.C.G.) is supported by the Belgian Foundation for Scientific Research (FNRS). This research was financed in part by FRFC grant 2-4507-82.

The Editor thanks S. Solomon and G. A. Victor for their assistance in evaluating this paper.

\section{REFERENCES}

Barth, C. A., and R. E Steele, Rocket observations of the NII 2143A dayglow, Geophys. Res. Lett., 9, 559, 1982.

Barth, C. A., D. W. Rusch, and A. I. Stewart, The UV nitric oxide experiment for Atmosphere Explorer, Radio Sci., 8, 379, 1973.

Cravens, T. E., The global distribution of nitric oxide at $200 \mathrm{~km}, J$. Geophys. Res., 86, 5710, 1981.

Cravens, T. E., J. C. Gérard and A. I. Stewart, and D. W. Rusch, The latitudinal gradient of nitric oxide in the lower thermosphere, $J$. Geophys. Res., 84, 2675, 1979.

Cravens, T. E., J. C. Gérard, M. Lecompte, A. I. Stewart, and D. W. Rusch, The global distribution of nitric oxide in the thermosphere as determined by the Atmosphere Explorer D satellite, J. Geophys. Res., $90,9862,1985$.

Dalgarno, A., G. A. Victor, and T. W. Hartquist, The auroral 2145 A feature, Geophys. Res. Lett., 8, 603, 1981.
Dick, K. A., The auroral 2150A feature: A contribution from lines of singly ionized nitrogen, Geophys. Res. Lett., 5, 273, 1978.

Duysinx, R., and A. Monfils, Auroral spectra recorded between $2000 \mathrm{~A}$ and $3000 \mathrm{~A}$ with a fast scanning spectrometer, Ann. Geophys., 28, $109,1972$.

Garcia, R., and S. Solomon, The effect of breaking gravity waves on the dynamics and chemical composition of the mesosphere and lower themosphere, J. Geophys. Res., 90, 3850, 1985.

Gérard, J. C., and E. J. Deneye, The transport of thermospheric nitric oxide into the mesosphere, Ann. Geophys., 2, 187, 1984.

Gérard, J. C., and R. G. Roble, The role of nitric oxide on the zonally averaged structure of the thermosphere: Solstice conditions for solar cycle minimum, Planet. Space Sci., 34, 131, 1986.

Gérard, J. C., R. G. Roble, D. W. Rusch, and A. I. Stewart, The global distribution of thermospheric odd nitrogen for solstice conditions during solar cycle minimum, J. Geophys. Res., 89, 1725, 1984.

Hedin, A. E., A revised thermospheric model based on mass spectrometer and incoherent scatter data: MSIS-83, J. Geophys. Res., $88,10,170,1983$.

Knight, R. D., Radiative lifetime of the ${ }^{5} S_{2}$ metastable state of $\mathrm{N}^{+}$, Phys. Rev. Lett., 48, 792, 1982.

Roble, R. G., and P. B. Hays, Density profile of atmospheric gases from planetary occultation data, Planet. Space Sci., 20, 1727, 1972.

Sharp, W. E., and M. H. Rees, Auroral spectrum between 1200 and 4000 angstroms, J. Geophys. Res., 77, 1810, 1972.

Stewart, A. I., and T. E. Cravens, Diurnal and seasonal effects in $E$ region low-latitude nitric oxide, J. Geophys. Res., 83, 2453, 1978.

Torr, M. R., and D. G. Torr, The NII 2143-A dayglow from Spacelab 1, J. Geophys. Res., 90, 6679, 1985a.

Torr, M. R., and D. G. Torr, Ionization frequencies for solar cycle 21: Revised, J. Geophys. Res., $90,6675,1985 b$.

Torr, M. R., D. G. Torr, R. A. Ong, and H. E. Hinteregger, Ionization frequencies for major thermospheric constituents as a function of solar cycle 21, Geophys. Res. Lett., 6, 671, 1979.

Victor, G. A., and A. Dalgarno, The 2145A dayglow and the high altitude nitric oxide density, Geophys. Res. Lett., 9, 866, 1982.

Wight, G. R., M. J. Van der Wiel, and C. E. Brion, Dipole excitation, iónization and fragmentation of $\mathrm{N}_{2}$ and $\mathrm{CO}$ in the $10-60 \mathrm{eV}$ region, J. Phys. B, 9, 675, 1976.

J. C. Gérard and C. E. Noël, Institut d'Astrophysique, Université de Liège, 5 avenue de Cointe, 4200 Liège-Ougrée, Belgium.

(Received October 30, 1985; revised May 15, 1986; accepted April 25, 1986.) 